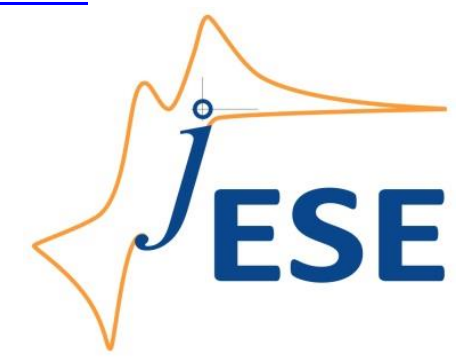

Open Access : : ISSN 1847-9286

www.jESE-online.org

Original scientific paper

\title{
Insights into electrochemical dealloying of Cu out of Au-doped Pt-alloy nanoparticles at the sub-nano-scale
}

\author{
Matija Gatalo ${ }^{1,2}$, Primož Jovanovič ${ }^{1}$, Francisco Ruiz-Zepeda ${ }^{1}$, Andraž Pavlišič ${ }^{3}$, \\ Ana Robba ${ }^{1,2}$, Marjan Bele ${ }^{1}$, Goran Dražić ${ }^{1}$, Miran Gaberšček ${ }^{1,2}$, Nejc Hodnik ${ }^{3, 凶}$ \\ ${ }^{1}$ Department of Materials Chemistry, National Institute of Chemistry, Hajdrihova 19, SI-1000 \\ Ljubljana, Slovenia \\ ${ }^{2}$ Faculty of Chemistry and Chemical Technology, University of Ljubljana, Vecna pot 113, SI-1000 \\ Ljubljana, Slovenia \\ ${ }^{3}$ Department of Catalysis and Chemical Reaction Engineering, National Institute of Chemistry, \\ Hajdrihova 19, SI-1000, Ljubljana, Slovenia
}

Corresponding authors E-mail: ${ }^{\bowtie}$ nejc.hodnik@ki.si; Tel.: +386 14760212

Received: December 6, 2017; Revised: December 30, 2017; Accepted: January 8, 2018

\begin{abstract}
Pt alloy nanoparticles present the most probable candidate to be used as the cathode cathodic oxygen reduction reaction electrocatalyst for achieving commercialization targets of the low-temperature fuel cells. It is therefore very important to understand its activation and degradation processes. Besides the ones known from the pure Pt electrocatalysts, the dealloying phenomena possess a great threat since the leached lessnoble metal can interact with the polymer membrane or even poison the electrocatalyst. In this study, we present a solution, supported by in-depth advance electrochemical characterization, on how to suppress the removal of $\mathrm{Cu}$ from the Pt alloy nanoparticles.
\end{abstract}

\section{Keywords}

Electrocatalysis; EFC-ICP-MS; IL-TEM; Pt alloy; ORR

\section{Introduction}

The motivation of scientist, in general, is to improve the life quality of people. Still our development and ability to live without a shortage of food, clean water, energy, etc. are dependent on raw materials resources and economics. However, on the other hand, it is limited by the pollution and negative climate changes. Since it has been already recognized that the pollution caused by humankind indeed affects the environment, it is now time to shift the balance towards the sustainable technologies and renewable energy. In the energy sector, this means replacement of fossil fuels by electricity coming from the sun and wind. Since this energy sources are intermittent, 
the current grand challenge is to store this clean energy to match the consumption demand. This is achieved by energy conversion like batteries, flow batteries, pump hydro, compressed air, flywheels, etc. or conversion to hydrogen via electrolyzers. Hydrogen can then be elegantly converted back to electricity via proton exchange membrane fuel cells (PEM FC) with only byproducts being water and heat [1-3]. This is very useful in the transportation and stationary sector.

In order to increase the PEM FC efficiency, Pt, as cathodic oxygen reduction reaction (ORR), is alloyed with less noble 3D metals like $\mathrm{Co}, \mathrm{Ni}$ and $\mathrm{Cu}$ [1,4-12]. Initial performances of Pt-alloys are already surpassing the US Department of Energy targets [1]. However, their stability is still under debate and is the topic of many current and future studies [13-20]. Three issues are foreseen for Ptalloys: (i) loss of initially superior ORR activity [13], (ii) formation of unstable porous nanoparticles $[9,15]$ and (iii) poisoning of the PEM FC by the leached less noble metal cations [12,21-23]. It is known that leached metals can cause the increase of the resistance of the polymer membrane and the lowering of the performance of the anode electrocatalysts due to the poisoning of the Pt surface $[12,23]$.

In this study, we have examined the effect of Au doping on dealloying of Cu out of highly active $\mathrm{PtCu}_{3}$ alloy nanoparticles supported on high surface area carbon (hereinafter referred to as Audoped $\mathrm{PtCu}_{3} / \mathrm{C}$ ). Although this effect is already known in the literature $[6,14,24-26]$, we provide additional insight into this very effective less noble metal removal suppression tactic. We utilize state-of-the-art advanced electrochemical techniques like thin film rotating disc electrode (TF-RDE), electrochemical flow cell coupled to inductively coupled plasma to mass spectroscopy (EFC-ICP-MS) and identical location (scanning) transmission electron microscopy (IL-(S)TEM).

\section{Experimental}

\section{Synthesis:}

The starting material $\mathrm{PtCu}_{3} / \mathrm{C}$ - intermetallic partially ordered (ordered shell, disordered core) $\mathrm{PtCu}_{3}$ nanoparticles that are tightly embedded (anchored) into modified carbon support were prepared via a modified sol-gel synthesis using a gelatin precursor $[5,14,17,27,28]$. Briefly, the synthesis consists of two vital steps, the first being annealing of a non-noble metal precursor (e.g., a $\mathrm{Cu}$ precursor) together with gelatine and carbon black to obtain embedded nanoparticles in a porous carbon matrix. In the second part, the $\mathrm{Cu}$ from the composite is partly galvanically replaced by a $\mathrm{Pt}$ precursor and annealed for the second time. The starting material was prepared in a $5 \mathrm{~g}$ batch.

From the starting material, we have then prepared two completely comparable materials which differ only in the small amount of noble metal addition ( $\mathrm{Au}$ or Pt). In both cases, $100 \mathrm{mg}$ of the starting material was dispersed in $500 \mathrm{~mL}$ of $5 \mathrm{mM} \mathrm{KCl}$ and purged with Ar for 1 hour while stirring in order to remove the oxygen from the suspension. Afterwards, while continuously purging the starting material suspension with $\mathrm{Ar}, 500 \mathrm{~mL}$ of $40 \mu \mathrm{M}$ solution of $\mathrm{Au}$ or Pt metal salt solution $\left(\mathrm{HAuCl}_{4}\right.$ or $\mathrm{K}_{2} \mathrm{PtCl}_{4}$. Approximately $4 \mathrm{mg}$ of $\mathrm{Au}$ or $\mathrm{Pt}$ ) was added drop-wise in a timeframe of 2 hours in order to galvanically displace superficial $\mathrm{Cu}$ atoms found at selected spots of the nanoparticles surface. Both noble metal decorated materials were then filtrated, re-dispersed in $0.1 \mathrm{M} \mathrm{KOH}$, filtrated again and re-dispersed 3 additional times in hot Mili-Q water followed by filtration. After the last filtration, the materials were dried overnight at $50^{\circ} \mathrm{C}$.

Both noble metal decorated materials were then placed together in the same furnace and subjected to an additional thermal annealing step at $500^{\circ} \mathrm{C}$ for 3 hours in a CO-containing atmosphere (two separate batches of annealing). Prior to thermal annealing in CO, the samples were first purged with Ar overnight to ensure an oxygen-free atmosphere. Furthermore, the 
samples were first heated to only $120^{\circ} \mathrm{C}$ for 1 hour in order to remove any residual moisture from both samples before moving to $500{ }^{\circ} \mathrm{C}$. Two materials were obtained - (i) Au-doped $\mathrm{PtCu} / \mathrm{C}$ and (ii) Pt-doped $\mathrm{PtCu}_{3} / \mathrm{C}$.

$X R D$

The powder X-ray diffraction (XRD) measurements of all samples were carried out on a Siemens D5000 diffractometer with Cu K 1 radiation $(\lambda=1.5406 \AA)$ in the $2 \theta$ range from $10^{\circ}$ to $60^{\circ}$ with the $0.04^{\circ}$ step per $1 \mathrm{~s}$. Samples were prepared on zero-background Si holder.

Electrochemical evaluation via Thin Film Rotating Disc Electrode (TF-RDE):

Electrochemical measurements were conducted in a two-compartment electrochemical cell in a $0.1 \mathrm{M} \mathrm{HClO}_{4}$ (Merck, Suprapur, $70 \%$ ) electrolyte with a conventional three-electrode system controlled by a potentiostat (Compact stat, Ivium technologies). $\mathrm{Ag} / \mathrm{AgCl}$ was used as a reference and a Pt wire as a counter electrode. The working electrode was a glassy carbon disk embedded in Teflon (Pine Instruments) with a geometric surface area of $0.196 \mathrm{~cm}^{2}$. Prior to any experiment, the two-compartment electrochemical cell was boiled in $18 \mathrm{M} \Omega \mathrm{cm}^{-1}$ Mili-Q water for 1 hour, and the electrode was polished to mirror finish with $\mathrm{Al}_{2} \mathrm{O}_{3}$ paste (particle size $0.05 \mu \mathrm{m}$, Buehler) on a polishing cloth (Buehler). After polishing, the electrodes were rinsed and sonicated in $18{\mathrm{M} \Omega \mathrm{cm}^{-1}}^{-1}$ Mili-Q water for 5 minutes. $20 \mu \mathrm{L}$ of $1 \mathrm{mg} \mathrm{mL}^{-1}$ water-based well-dispersed catalysts ink was pipetted on the glassy carbon electrode completely covering it and dried under ambient conditions. Such preparation resulted in the loading of approximately $25 \mu \mathrm{g}_{\mathrm{Pt}} \mathrm{cm}^{-2}$ and was found to be optimal for obtaining high specific activities at $0.9 \mathrm{~V}_{\text {RHEE }}$. After drying, the electrode was mounted on the rotator (Pine Instruments). The electrode was placed in Ar saturated electrolyte under potential control at $0.05 \mathrm{~V}_{\mathrm{RHE}}$. All electrocatalysts were electrochemically activated for 200 cycles between 0.05 and $1.35 \mathrm{~V}_{\mathrm{RHE}}$ with a scan rate of $300 \mathrm{mV} \mathrm{s}^{-1}$. Degradation measurements were performed after electrochemical activation, each time with a fresh thin film of the electrocatalyst material. The materials were degraded for 10000 cycles between 0.4 and 1. $x V_{R H E}(x=0,2,4)$ and for 50000 cycles between $0.4-1.0 V_{\text {RHE. }}$. After electrochemical activation and after degradation we exchanged the electrolyte and measured both CO stripping and ORR polarization curve. ORR polarization curves were measured in an oxygen saturated electrolyte with rotation at 1600 RPM in the same potential window with a scan rate of $20 \mathrm{mV} \mathrm{s}^{-1}$. After subtraction of background current due to capacitive currents, kinetic parameters were calculated at $0.9 \mathrm{~V}_{\mathrm{RHE}}$. Ohmic resistance of the electrolyte was determined and compensated for as reported in [29] Electrochemical surface area (ESA) was determined by integrating the charge in $\mathrm{CO}$ stripping experiments as described in [30]. All potentials are given against the reversible hydrogen electrode (RHE), which was measured before the start of the experiment and at the end. At the end of each experiment, the thin film on the RDE was dispersed in $0.5 \mathrm{~mL}$ of isopropanol with the help of an ultrasound bath and later deposited on a TEM grid for ex-situ TEM analysis or wiped from the RDE with a carbon conductive adhesive tapes for SEM-EDX analysis.

Scanning Electron Microscopy: Energy Dispersion X-ray spectrometry (SEM-EDX):

Ex-situ SEM-EDX analysis was carried out with SUPRA 35 VP (Carl Zeiss) that is equipped with Energy Dispersion X-ray Spectrometer Inca 400 (Oxford Instruments). The operational voltage was set to $20 \mathrm{kV}$, the distance between the gun and the sample was set to $8.5 \mathrm{~mm}$ for optimal signal acquisition and areas for SEM-EDX analysis were selected under magnification of 20000 . For statistical relevance, 3 different areas were measured for each sample. 


\section{Transmission Electron Microscopy (TEM):}

Transmission Electron Microscopy was carried out in a probe Cs-corrected scanning transmission electron microscope Jeol ARM 200 CF equipped with an SDD Jeol Centurio Energy-dispersive X-ray (EDX) spectrometer. The operation voltage was set to $200 \mathrm{kV}$. High Angle Annular Dark Field (HAADF) images were taken with 68 and 180 mrad for inner and outer semiangles. Convergence angle was set to 25 mrads.

\section{Identical Location Transmission Electron Microscopy (IL-TEM)):}

The electrocatalyst suspension $\left(1 \mathrm{mg} \mathrm{mL}^{-1}\right)$ was ultrasonicated for 15 minutes and diluted 10 times (100 uL of the suspension, $900 \mathrm{uL}$ of Mili-Q water). After additional 5 minutes of ultrasonication (diluted suspension), $5 \mathrm{uL}$ of the suspension was deposited on Au-grid (Plano Au finder grid (NH7) coated with a Lacey carbon film). Once dried, the grid was inspected under TEM. Several spots were registered in the finder grid under TEM and STEM. Locations of the spots were recorded at different magnifications to facilitate the afterward tracking. EDX maps and line scans were performed spending the minimum time to avoid any possible beam damage. The grid was afterward removed from the microscope to perform the electrochemical tests as following. After the inspection, the grid was mounted on a glassy carbon disc, embedded in Teflon (Pine Instruments) with a geometric surface area of $0.196 \mathrm{~cm}^{2}$. Electrochemical activation was conducted in a two-compartment electrochemical cell in a $0.1 \mathrm{M} \mathrm{HClO}_{4}$ (Merck, Suprapur, $70 \%$ ) electrolyte with a conventional three-electrode system controlled by a potentiostat (Compact stat, Ivium technologies). The electrode was mounted on the rotator (Pine Instruments). The electrode was placed in Ar saturated electrolyte under potential control at $0.05 \mathrm{~V}$ ( $v s$. RHE). The electrocatalyst on the Au-grid was electrochemically activated for 200 cycles between 0.05 and $1.35 V_{\text {RHE }}$ with a scan rate of $300 \mathrm{mV} \mathrm{s}^{-1}$. All potentials are given against the reversible hydrogen electrode (RHE). After electrochemical activation, the Au-grid was dipped into fresh Mili-Q water and left to dry at room temperature. Once dried, the gird was once again inspected under TEM and STEM. IL was identified by the low magnification images which hold marked locations of previously analyzed areas on the TEM grid labeled with letters. The images and the EDX spectra are then taken under the same conditions as previously.

\section{Electrochemical flow cell coupled to ICP-MS (EFC ICP-MS)}

A replica of BASi electrochemical flow cell (Cross-Flow Cell Kit MW-5052) made of TECAPEEK material was coupled with an Agilent 7500ce ICP-MS instrument (Agilent Technologies,Palo Alto, USA) equipped with a MicroMist glass concentric nebulizer and a Peltier-cooled Scott-type doublepass quartz spray chamber. A forward radio frequency power of $1500 \mathrm{~W}$ was used with the following Ar gas flows: carrier $0.85 \mathrm{~L} \mathrm{~min}^{-1}$, makeup $0.28 \mathrm{~L} \mathrm{~min}^{-1}$, plasma $1 \mathrm{~L} \mathrm{~min}^{-1}$, and cooling $15 \mathrm{~L} \mathrm{~min}$. $\mathrm{HClO}_{4}\left(0.1 \mathrm{~mol} \mathrm{~L}^{-1}\right.$, Merck, Suprapur, $70 \%$ ) carrier solution for the electrochemical experiments was pumped at $263 \mathrm{~L} \mathrm{~min}^{-1}$ by using a peristaltic pump into the BASi EC flow cell and then directly into the nebulizer of the ICP-MS. The carrier solution and electrochemical cell were electrically grounded with respect to the ICP-MS to minimize the possibility of ICP-MS response spikes from static charging at the peristaltic pump rollers. The ICP-MS was tuned for high sensitivity to obtain the best possible signal-to-noise ratio for the measurements. The concentration of catalyst suspension was set to $1 \mathrm{mg} \mathrm{mL}^{-1}$. The suspension was drop-casted by micropipette over one of the GC electrodes and stabilized by a $5 \mu \mathrm{L}$ of $\mathrm{Nafion}^{\circledR}$ ( 5 wt.\% water suspension) diluted by isopropanol (1/50). Second GC electrode was used as a counter electrode. The orientation of the working electrode (WE) and counter electrode (CE) was adjusted so that WE was placed after CE in direction of the electrolyte 
flow. An electrochemical protocol performed consisted of 200 cycles $\left(300 \mathrm{mV} \mathrm{s}^{-1}\right)$ between 0.05 and $1.35 \mathrm{~V}_{\text {RHE. }}$

\section{Monte Carlo simulations}

Kinetic Monte Carlo (KMC) modeling was performed by simply applying Arrhenius equation for the overall bond energy of the atom (first: activation energy is depended on bond type; second: the overall bond energy of the atom is rising with number of neighbors). In the iterative loop atom was randomly chosen to perform specific process (dissolution or diffusion) which corresponds to updated crystal mesh. The loop was running until the specific dealloying time was achieved (for more details look in ref. "Atomically resolved dealloying of structurally ordered Pt nanoalloy as oxygen reduction reaction electrocatalyst"). By varying initial chemical compositions, atoms distribution and shape/size of the nanoparticles, obtained by TEM and EDX observations, KMC simulations resulted in different morphological changes.

\section{Results and discussion}

We prepared three $\mathrm{PtCu}_{3} / \mathrm{C}$ analogs: (i) the starting or as-prepared $\mathrm{PtCu}_{3} / \mathrm{C}$, (ii) Pt-doped $\mathrm{PtCu}_{3} / \mathrm{C}$ and (iii) Au-doped $\mathrm{PtCu}_{3} / \mathrm{C}$. For the preparation of the last two samples, the first one was used as a precursor. This means that all samples have the same particle size distribution. Since Pt and Au are more noble than $\mathrm{Cu}$ [31] the galvanic displacement can occur. Therefore, some surface superficial $\mathrm{Cu}$ is replaced by $\mathrm{Pt}$ and in the last sample by Au cations form the solution. This results in the removal of less than $1 \%$ of $\mathrm{Cu}$. The additional annealing step is needed for the formation of the Pt or PtAu skin [14]. For this reason, we can also consider that the composition of the three samples is very similar. The idea behind doping of $\mathrm{PtCu}_{3} / \mathrm{C}$ nanoparticles skin was to study the effects caused by very small additions of $\mathrm{Au}$, and also Pt as a reference point.

First cyclic voltammograms (Fig. 1a), which is a part of the electrochemical activation (EA) protocol of 200 cycles, $300 \mathrm{mV} \mathrm{s}^{-1}, 0.05-1.35 \mathrm{~V}_{\mathrm{RHE}}$, performed with TF-RDE shows comparison of $\mathrm{Cu}$ dealloying in the first cycle of the EA. By measuring the charge obtained in the first cycle of EA (Fig. 1a), we notice that in the case of both $\mathrm{Au}$ and Pt decoration, the total amount of dealloyed $\mathrm{Cu}$ is smaller compared to the as-prepared $\mathrm{PtCu}_{3} / \mathrm{C}$. This is a direct proof of successful galvanic displacement of superficial $\mathrm{Cu}$. The success of galvanic displacement is further confirmed by the presence of an additional Au peak at approximately $38.5^{\circ} 2 \theta$ (Fig. 1c, dash blue), while we cannot observe any additional peaks in the case of Pt decoration which points towards too small size of the Pt clusters (Fig. 1b, dash red). Surprisingly, there is a visible difference in the intensity of both pm $3 \mathrm{~m}$ peaks ( $\mathrm{PtCu}_{3}$ ordered phase) upon decorating with both $\mathrm{Pt}$ and $\mathrm{Au}$. After annealing, Au doping is shown to have an effect on the bulk structure of the $\mathrm{PtCu}_{3}$ nanoparticles by further decreases the intensity of the peaks corresponding to the pm $3 \mathrm{~m}$ phase, while also slightly broadening the peaks corresponding to the $\mathrm{fm} 3 \mathrm{~m}$ phase (Fig 1c, blue). The phenomena could be directly correlated to the doping process itself, as it could point towards Au acting as an impurity in the crystal lattice of the $\mathrm{PtCu}_{3}$ nanoparticles and consequently decreasing the average size of a domain. One can also notice that the peak for pure Au disappeared, while there is an appearance of weak new peaks that most likely correspond to the small number of small-ternary nanoparticles. The same phenomena cannot be noticed in the case of Pt doping, where we don't observe any major changes to the crystal lattice upon annealing (Fig. 1C, red). Although we are dealing with very similar size distribution, we observe a huge difference in the case of the electrochemically active surface area (ESA) (Fig. 1d). This is due to the decreased degree of porosity in the case of the Au-doped $\mathrm{PtCu}_{3} / \mathrm{C}$ analog [25]. 
a)

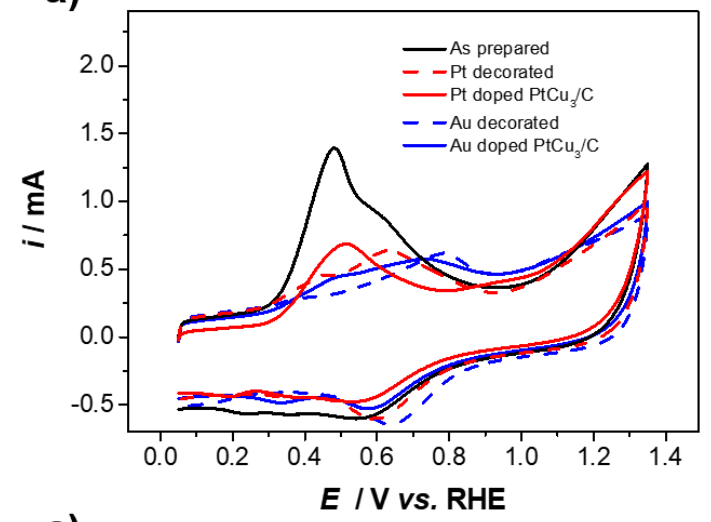

c)

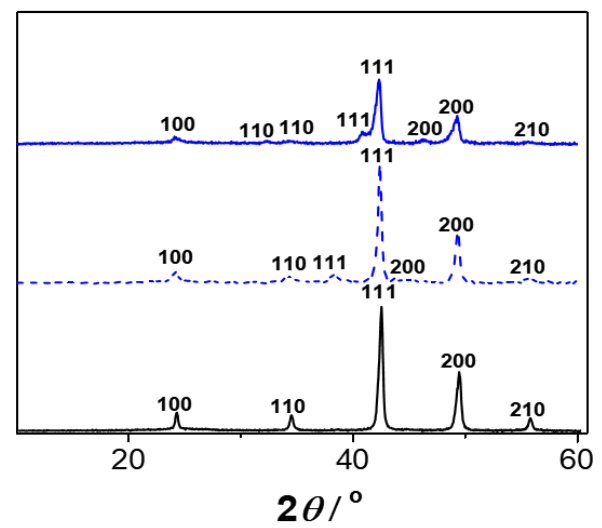

b)

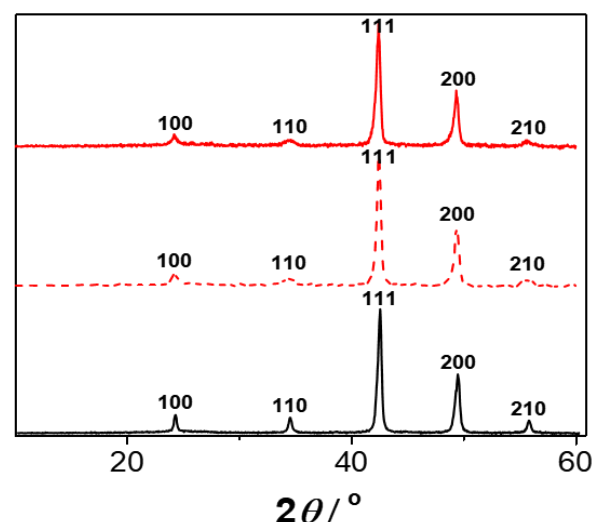

d)

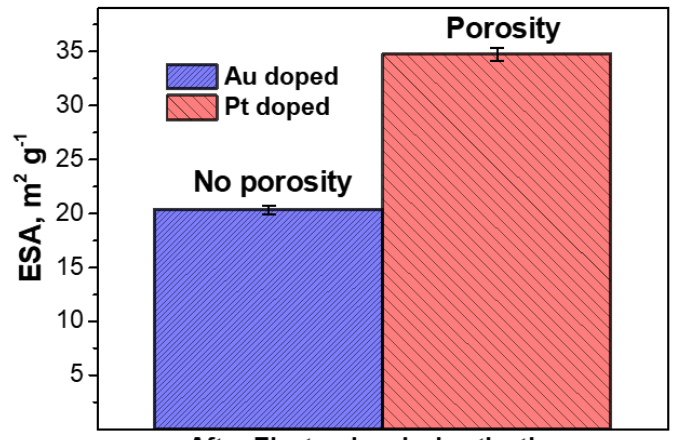

After Electrochemical activation, 200 cycles, $300 \mathrm{mV} \mathrm{s}^{-1}, 0.05-1.35 \mathrm{~V}$ vs. RHE

Figure 1. (a) first cyclic voltammogram of EA of (i) as-prepared $\mathrm{PtCu} / \mathrm{C}$, (ii) decorated $\mathrm{PtCu}_{3} / \mathrm{C}$ and doped $\mathrm{PtCu}_{3} / \mathrm{C}$ analogs, (b) and (c) powder XRD comparison (colors and patterns match the legend in figure a) and

(d) ESA measurement (from CO stripping) after EA of (i) Au-doped PtCu$/ \mathrm{C}$ and (ii) Pt-doped $\mathrm{PtCu}_{3} / \mathrm{C}$.

Formation of porous nanoparticles in the case of $\mathrm{Pt}$-doped $\mathrm{PtCu}_{3} / \mathrm{C}$ analog results in the increase in the ESA. This surface area is, however, not stable due to the coarsening of the porous structure even at mild degradation simulations till $1 \mathrm{~V}_{\mathrm{RHE}}$ [15]. This is nicely seen from the Fig. 2 where ESA of both analogs after degradation is limiting towards a similar value. Fig. 3a shows the drop of the specific activity of both doped analogs. Au-doped $\mathrm{PtCu}_{3} / \mathrm{C}$ analog exhibits about $25 \%$ lower initial specific activity. We presume that this is due to the increased activity of the porous nanoparticles on account of the confinement effect [32]. However, as can be seen in the same figure, the specific activity is decreasing faster for the Pt-doped sample. This is because of the increased loss of $\mathrm{Cu}$ and the fact that porous particles are not stable [15]. The trend indicates that further degradation would result in the faster loss of specific activity, as well as ESA of the Pt-doped $\mathrm{PtCu}_{3} / \mathrm{C}$ analog.

Removal of the $\mathrm{Cu}$ from the two doped $\mathrm{PtCu}_{3}$ electrocatalysts was measured by energy-dispersive X-ray spectroscopy detector in the SEM (SEM-EDX). In Fig. 4 it is clearly seen that Au-doped $\mathrm{PtCu}_{3} / \mathrm{C}$ analog losses much less $\mathrm{Cu}$ upon EA and also upon all follow-up degradation protocols. EFC-ICP-MS measurements (Fig. 5a) directly prove the enhanced retention of $\mathrm{Cu}$ from the $\mathrm{Au}$-doped $\mathrm{PtCu}_{3} / \mathrm{C}$ analog. Leached Cu cations present a serious threat to the real application of PEM FC. It can increase the internal resistance of polymer membrane and can also poison the electrocatalyst active $\mathrm{Pt}$ surface [1-4]. And on the other hand, retention of $\mathrm{Cu}$ is essential to sustain enhanced ORR activity via ligand and/or strain effects [13]. The negative effect of $\mathrm{Cu}$ dealloying under long-term PEM FC operating conditions (e.g., simulated via degradation protocol of 50000 cycles, $0.4-1.0 \mathrm{~V}_{\mathrm{RHE}}, 1 \mathrm{~V} \mathrm{~s}$ $\left.{ }^{1}\right)$ can be visible in the change of the slope of ORR polarization curve (Fig. $5 \mathrm{~b}$ ) in the case of Pt-doped $\mathrm{PtCu}_{3} / \mathrm{C}$ analog. 
a)

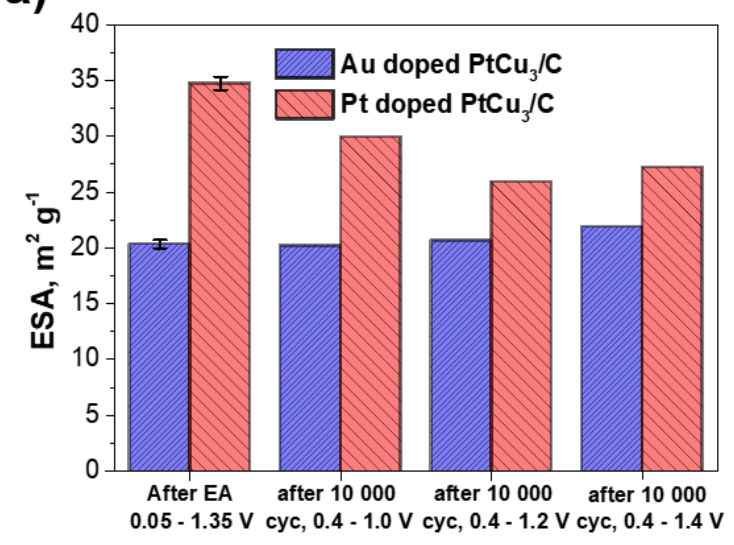

b)

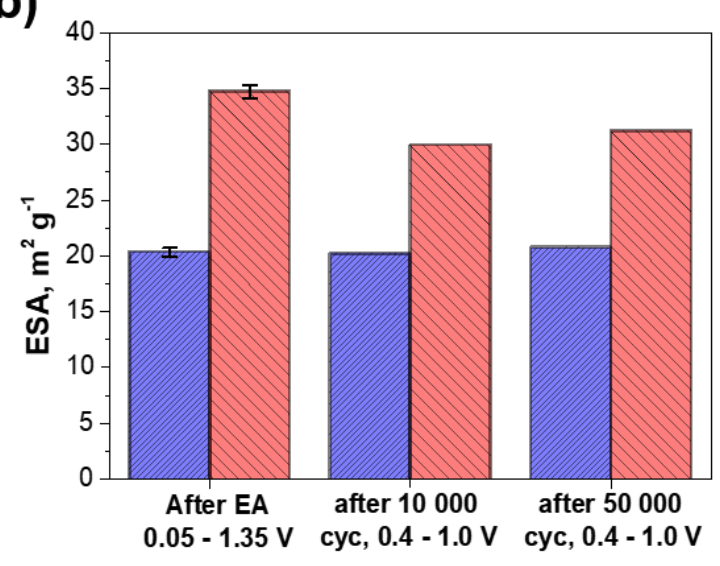

Figure 2. The absolute decrease of ESA of the two doped PtCu analogs: Au-doped PtCu $/ C$ and Pt-doped $\mathrm{PtCu}_{3} / \mathrm{C}$ upon different degradation protocols. (a) Comparison of ESA for both analogs when varying the upper potential limit of the degradation protocol $\left(0.05-1 . X V_{R H E} X=0,2,4\right)$, while keeping number of cycles constant and (b) comparison of both analogs when varying the number of cycles (10 000 and 50000$)$,

while keeping the upper potential limit of the degradation protocol constant. Both analogs were electrochemically activated prior to each degradation protocol.

Such a change in slope can be explained by the redeposition of dealloyed and subsequently underpotentially deposited $\mathrm{Cu}$ ( $\mathrm{Cu}$ upD) [21]. Furthermore, when comparing $\mathrm{CO}$ stripping of Pt-doped $\mathrm{PtCu}_{3} / \mathrm{C}$, a slight but clear decrease in ESA is observed (Fig. $5 \mathrm{c}, \mathrm{d}$ ). That further proves the sintering of the pores even under mild degradation conditions. On the other hand, the slope of ORR polarization curve in the case of Au-doped $\mathrm{PtCu}_{3} / \mathrm{C}$ analog hasn't changed (Fig $5 \mathrm{~b}$ ). This further proves the benefit of higher Cu retention. Additionally, full ESA has been retained (Fig. $5 \mathrm{~d}$ ) in comparison to the CO stripping experiment after EA (Fig. 5C).

a)

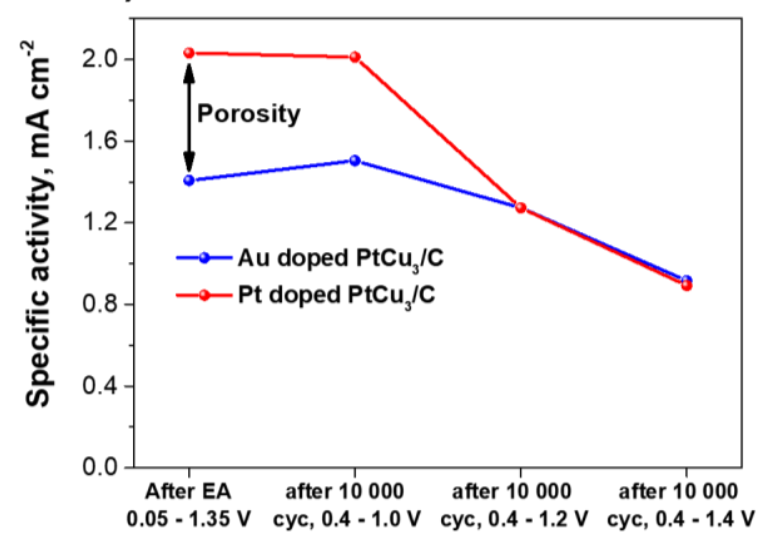

b)

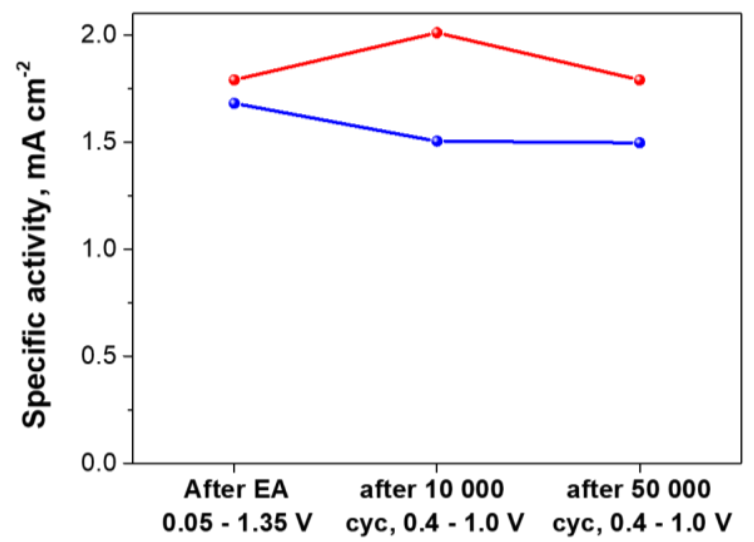

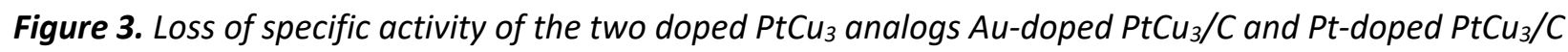
upon different degradation protocols. (a) Shows comparison of specific activity for both analogs when varying the upper potential limit of the degradation protocol $\left(0.05-1 . X V_{R H E} X=0,2,4\right)$, while keeping number of cycles constant and (b) shows comparison of both analogs when varying the number of cycles (10 000 and 50000$)$, while keeping the upper potential limit of the degradation protocol constant. Both analogs were electrochemically activated prior to each degradation protocol. 
a)

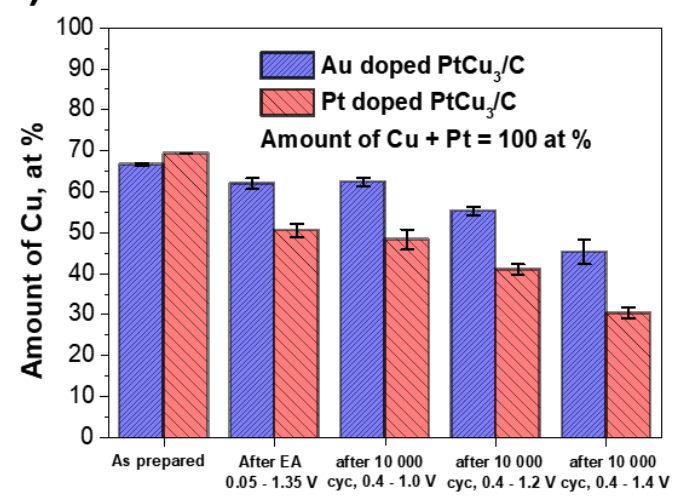

b)

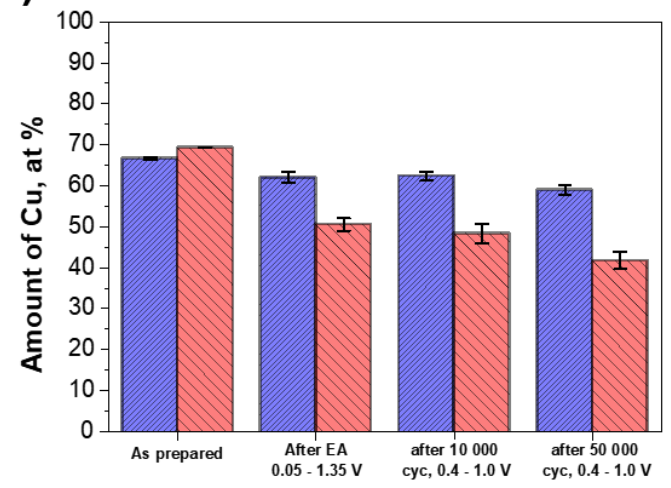

Figure 4. SEM-EDX measurements of Cu loss, \% of the two doped PtCu analogs: Au-doped $\mathrm{PtCu} / \mathrm{C}$ and Ptdoped $\mathrm{PtCu}_{3} / \mathrm{C}$ upon different degradation protocols. (a) Shows comparison of $\mathrm{Cu}$ loss, $\%$ for both analogs when varying the upper potential limit of the degradation protocol $\left(0.05-1 . X V_{R H E}, X=0,2,4\right)$, while keeping number of cycles constant and (b) shows comparison of both analogs when varying the number of cycles (10 000 and 50000$)$, while keeping the upper potential limit of the degradation protocol constant.

Both analogs were electrochemically activated prior to each degradation protocol.

a)
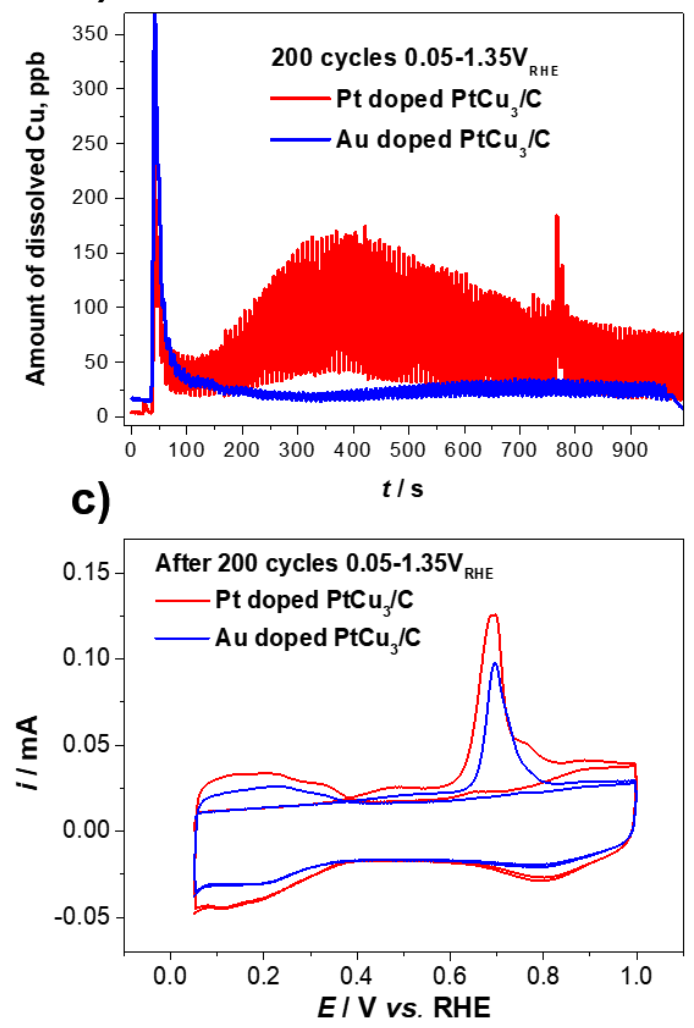

b)

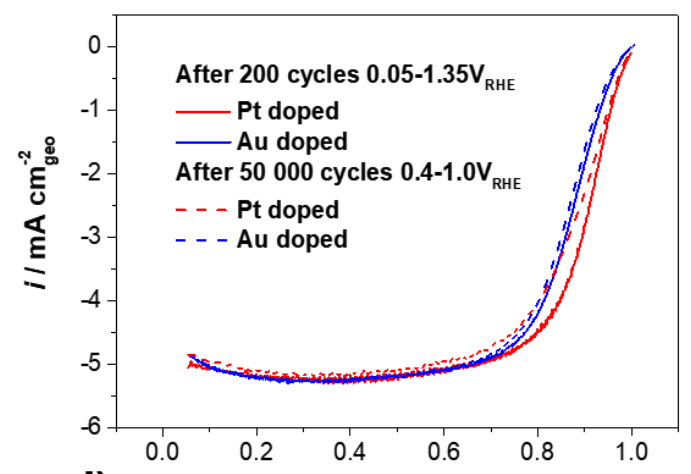

d)

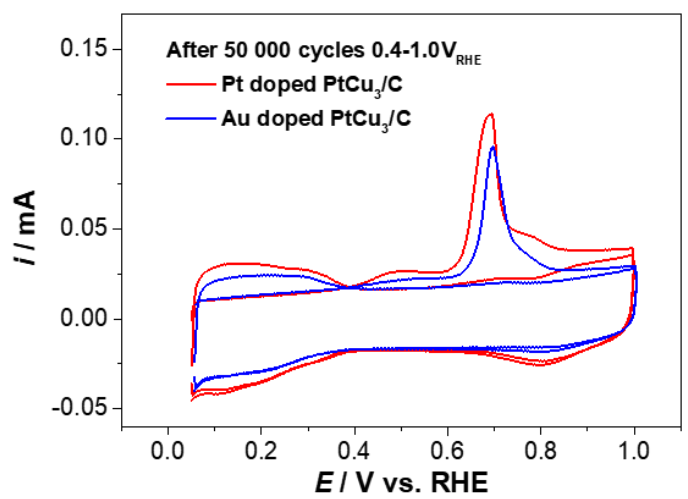

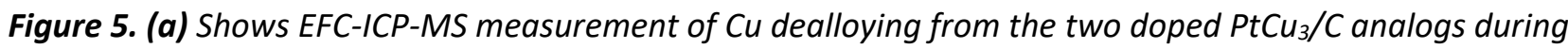
200 cycles of EA ( $\left.300 \mathrm{mV} / \mathrm{s}, 0.05-1.35 \mathrm{~V}_{\mathrm{RHE}}\right)$. (b) Shows polarization ORR curves after both the activation and degradation experiment in $\mathrm{O}_{2}$ saturated electrolyte $\left(0.1 \mathrm{M} \mathrm{HClO}_{4}\right)$. CO stripping of Pt \& Au-doped $\mathrm{PtCU}_{3} / \mathrm{C}$ electrocatalysts after (c) 200 cycles of EA $\left(0.05-1.35 \mathrm{~V}_{\mathrm{RHE}}, 300 \mathrm{mV} \mathrm{s}^{-1}\right)$ and after (d) $50000 \mathrm{cycles}$ of degradation $\left(0.4-1.0 \mathrm{~V}_{\text {RHE }} 1 \mathrm{~V} \mathrm{~s}^{-1}\right)$.

Transmission electron microscopy investigation confirms the results above on the sub-nanoscale. Pt-doped $\mathrm{PtCu}_{3} / \mathrm{C}$ analog exhibits extensive porosity formation and shrinkage in diameter as a result of $\mathrm{Cu}$ dealloying. In Fig. 6 IL-STEM HAADF images of the same Pt-doped nanoparticle before and after activation dealloying is shown. Line scan EDX profiles clearly confirm the formation of 
pores. Careful observation of the Pt line profile (red curve in Fig. 6) reveals the formation of the Pt surface enrichment. Kinetic Monte-Carlo simulations of dealloying process of the same size $\mathrm{PtCu}_{3}$ nanoparticle are in fascinating accordance with the IL-STEM images. As an input parameter, the $\mathrm{PtCu}_{3}$ nanoparticle already had two monolayers of $\mathrm{Pt}$ on the surface. If either none, one or three monolayers were chosen, results did not resemble the ones observed in the IL-STEM images [25]. For this reason, we presume that as-synthesized Pt-doped sample has, on average, already 2 monolayers of $\mathrm{Pt}$ on the surface. This atomistic insight into $\mathrm{PtCu}_{3}$ dealloying was only possible due to the coupling of the advanced characterization techniques with computer simulations.
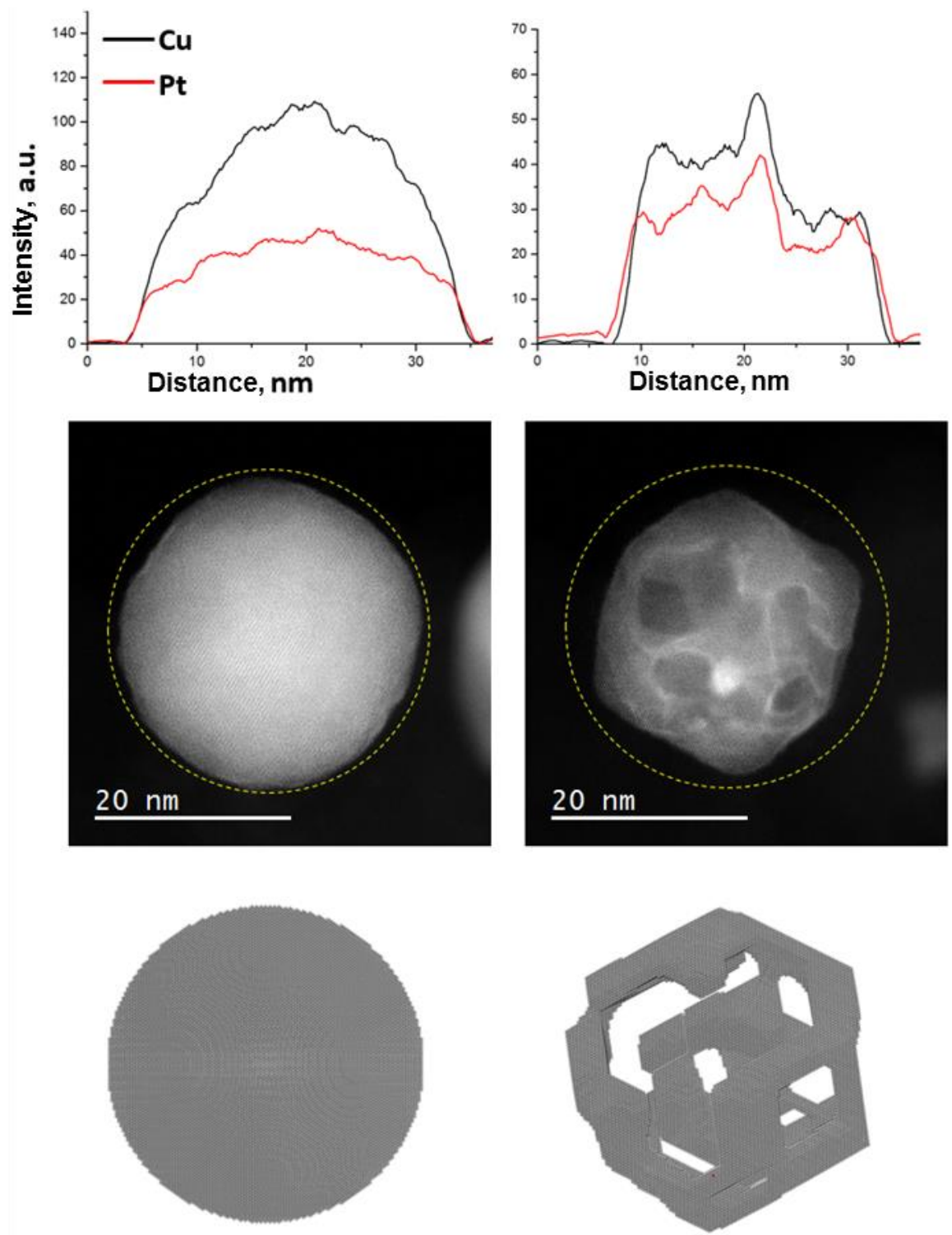

Figure 6. IL-STEM EDX line scan (top), HAADF image (middle) and Kinetic Monte Carlo simulations (bottom) of the same Pt-doped PtCu electrocatalyst before and after EA. An increase in the thickness of the Pt skin is observed after EA, additionally, faceting and pore formation are observed.

For the Au-doped $\mathrm{PtCu}_{3} / \mathrm{C}$ analog no porosity is observed (Fig. 7). This is in complete accordance with the previous results showing much lower Cu removal. Careful inspection of the IL-STEM HAADF (yellow dot circle around the particle in Fig. 7) also reveals small shrinkage and the formation of facets [13]. EDX Cu line profile indicates that negligible amount of $\mathrm{Cu}$ was removed at the activation protocol, unlike in the case of the Pt-doped sample. Again, the formation of Pt or probably PtAu skin is evident. Monte-Carlo simulation indicates that 4 monolayers of noble atoms (Pt and $\mathrm{Au}$ ) are present on the surface. As an approximation, here the surface diffusivity of Au and Pt were chosen 
to be the same. However, it is known from the literature that Au surface mobility is faster compared to Pt and has, therefore, better ability to "heal" the surface [33]. Therefore it is less probable that the porosity formation takes place even with the same thickness of pure Pt layer [17]. The formation of the facets is also seen in the Monte Carlo simulation.
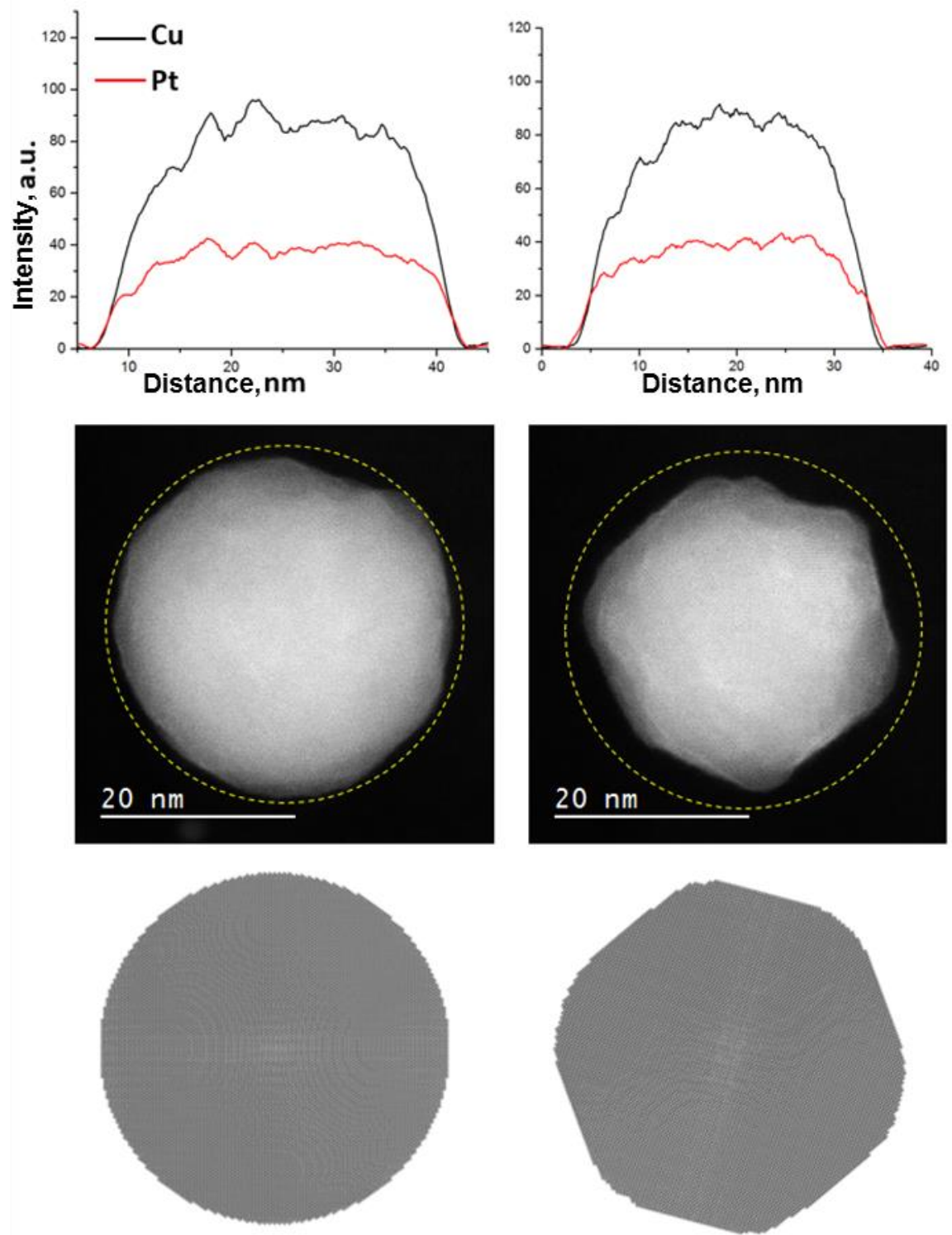

Figure 7. IL-STEM EDX line scan (top), HAADF image (middle) and Kinetic Monte Carlo simulations (bottom) of the same Au-doped PtCu electrocatalyst before and after electrochemical activation. An increase in the thickness of the Pt skin is observed after EA, but only faceting and no pore formation is observed.

The formation of the PtAu skin is also observed in the IL-STEM and IL-EDX elemental map images of the same Au-doped $\mathrm{PtCu}_{3}$ nanoparticles before and after electrochemical activation protocol in Fig. 8. In the IL-STEM images, the formation of more defined edges and also facets is seen (i.e., faceting) $[13,16]$. This is in accordance with Fig. 7. In IL-STEM HAADF the intensity of the edges is brighter, which indicates the presence of the metal with higher $Z$ like Pt and Au. This is confirmed by the IL-EDX elemental map. Careful observation also reveals the enrichment of the Pt on the surface even before the dealloying. This is in accordance with the Monte Carlo simulation results in Fig. 7. 

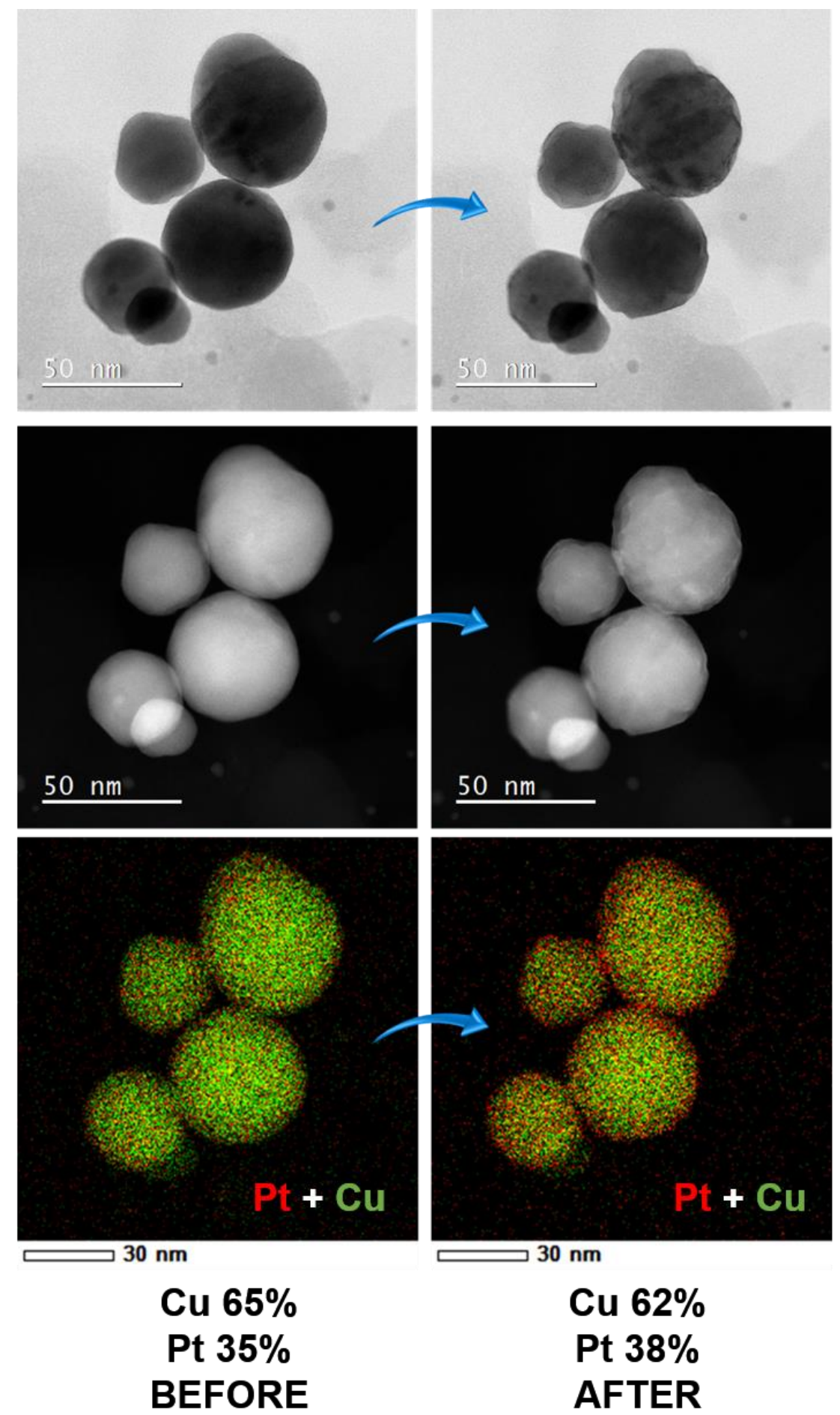

\section{Cu $62 \%$}

Pt 38\% AFTER

Figure 8. IL-STEM Bright Field (top), IL-STEM High Angle Annular Dark Field (middle) and EDX elemental mapping (bottom) of the same Au-doped $\mathrm{PtCu}_{3}$ electrocatalyst before and after $\mathrm{EA}$. Notice the etching of the surface in the particles and the increase of Pt skin thickness after EA.

In the Fig. 9 the exact atomic composition of the representative activated $\mathrm{Au}$-doped $\mathrm{PtCu}_{3}$ nanoparticle is measured with longer EDX elemental mapping and EDX line scan exposure. This was, however, avoided in the IL-EDX experiments to prevent alteration or damage of the nanoparticles structure by the electron beam. This is also the reason why Au signal could not be distinguished from the dominant Pt signal. Au is distributed over the entire crystal lattice of the nanoparticle. However, it can be presumed from the flat shape [34] of the Au line scan (Fig. 9c) that the concentration of Au lowers when moving from the surface towards the core. If Au was uniformly 
distributed, Au line scan would resemble the shape of the Cu scan. We can also distinguish that the surface is formed from at least 5 monolayers of PtAu skin (Fig. 9b).
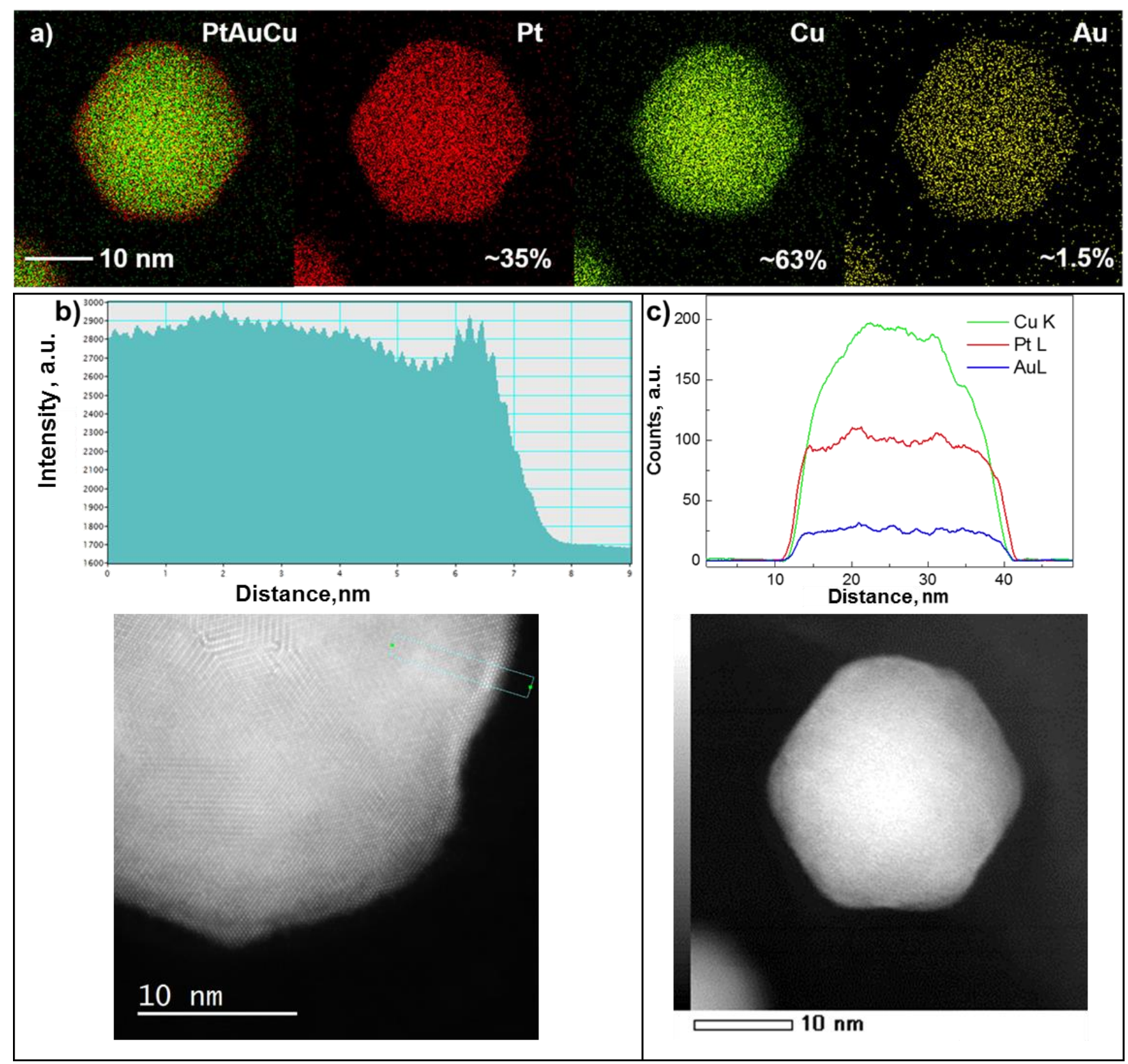

Figure 9. (a) EDX elemental mapping, (b) HAADF intensity line profile across particle and (c) line elemental scans of the Au-doped PtCu dealloyed nanoparticle.

\section{Conclusions}

Two completely comparable materials which differ only in the small amount of noble metal addition (Au or $\mathrm{Pt}$ ) have been prepared from the initial $5 \mathrm{~g}$ bath of highly active intermetallic ordered $\mathrm{PtCu}_{3} / \mathrm{C}$ electrocatalyst. In contrast to Pt doping (specific activity $\approx 2 \mathrm{~mA} \mathrm{~cm}{ }^{-2} \mathrm{Pt}$ ), Au doping of the $\mathrm{PtCu}_{3} / \mathrm{C}$ electrocatalyst results in approximately $25 \%$ lower initial specific activity, as well as lower ESA due to inhibition of porosity formation during 200 cycles of EA $\left(0.05-1.35 \mathrm{~V}_{\text {RHE }}, 300 \mathrm{mV} \mathrm{s}^{-1}\right)$. However, upon the degradation till 1.2 $\mathrm{V}_{\mathrm{RHE}}$ or higher, the difference between both Pt and Au-doped $\mathrm{PtCu}_{3} / \mathrm{C}$ analogs becomes negligible because Au-doped $\mathrm{PtCu}_{3} / \mathrm{C}$ analog exhibits much better stability, or in other words much lower stability of Pt-doped $\mathrm{PtCu}_{3} / \mathrm{C}$ analog. The increased stability of $\mathrm{Au}$-doped $\mathrm{PtCu}_{3} / \mathrm{C}$ analog is due to the prevention of the porosity formation and due to higher $\mathrm{Cu}$ retention. The most impressive result of the current study is the much-improved retention of $\mathrm{Cu}$ that presents potentially serious issues in the real PEM FC applications. 
Acknowledgements: Authors gratefully acknowledge the financial support of the Slovenian Research Agency (ARRS) through the Research Core Funding Programme P2-0152, P2-0393 and Project Z2-8161.

\section{References}

[1] M. K. Debe, Nature 486 (2012) 43-51.

[2] N. M. Markovic, Angewandte Chemie International Edition 53 (2014) 102-121.

[3] H. A. Gasteiger, S. S. Kocha, B. Sompalli, F. T. Wagner, Applied Catalysis B: Environmental 56 (2005) 935.

[4] M. Bele, P. Jovanovic, A. Pavlisic, B. Jozinovic, M. Zorko, A. Recnik, E. Chernyshova, S. Hocevar, N. Hodnik, M. Gaberscek, Chemical Communications 50 (2014) 13124-13126.

[5] C. Chen, Y. Kang, Z. Huo, Z. Zhu, W. Huang, H. L. Xin, J. D. Snyder, D. Li, J. A. Herron, M. Mavrikakis, M. Chi, K. L. More, Y. Li, N. M. Markovic, G. A. Somorjai, P. Yang, V. R. Stamenkovic, Science 343 (2014) 1339-1343.

[6] Z. W. Seh, J. Kibsgaard, C. F. Dickens, I. Chorkendorff, J. K. Nørskov, T. F. Jaramillo, Science 355 (2017).

[7] B. Han, C. E. Carlton, A. Kongkanand, R. S. Kukreja, B. R. Theobald, L. Gan, R. O'Malley, P. Strasser, F. T. Wagner, Y. Shao-Horn, Energy \& Environmental Science 8 (2015) 258-266.

[8] L. Gan, M. Heggen, R. O'Malley, B. Theobald, P. Strasser, Nano Letters 13 (2013) 1131-1138.

[9] A. S. Aricò, P. Bruce, B. Scrosati, J.-M. Tarascon, W. van Schalkwijk, Nature Materials 4 (2005) 366.

[10] D. Wang, Y. Yu, J. Zhu, S. Liu, D. A. Muller, H. D. Abruña, Nano Letters 15 (2015) 1343-1348.

[11] I. E. L. Stephens, A. S. Bondarenko, U. Gronbjerg, J. Rossmeisl, I. Chorkendorff, Energy \& Environmental Science 5 (2012) 6744-6762.

[12] N. Hodnik, C. Jeyabharathi, J. C. Meier, A. Kostka, K. L. Phani, A. Recnik, M. Bele, S. Hocevar, M. Gaberscek, K. J. J. Mayrhofer, Physical Chemistry Chemical Physics 16 (2014) 13610-13615.

[13] M. Gatalo, P. Jovanovič, G. Polymeros, J.-P. Grote, A. Pavlišič, F. Ruiz- Zepeda, V. S. Šelih, M. Šala, S. Hočevar, M. Bele, K. J. J. Mayrhofer, N. Hodnik, M. Gaberšček, ACS Catalysis 6 (2016) 1630-1634.

[14] C. Baldizzone, L. Gan, N. Hodnik, G. P. Keeley, A. Kostka, M. Heggen, P. Strasser, K. J. J. Mayrhofer, ACS Catalysis 5 (2015) 5000-5007.

[15] C. Jeyabharathi, N. Hodnik, C. Baldizzone, J. C. Meier, M. Heggen, K. L. N. Phani, M. Bele, M. Zorko, S. Hocevar, K. J. J. Mayrhofer, ChemCatChem 5 (2013) 2627-2635.

[16] N. Hodnik, M. Zorko, M. Bele, S. Hočevar, M. Gaberšček, The Journal of Physical Chemistry C 116 (2012) 21326-21333.

[17] M. Zorko, B. Jozinović, M. Bele, N. Hodnik, M. Gaberšček, Ultramicroscopy 140 (2014) 44-50.

[18] M. Oezaslan, M. Heggen, P. Strasser, Journal of the American Chemical Society 134 (2012) 514-524.

[19] N. Hodnik, G. Dehm, K. J. J. Mayrhofer, Accounts of Chemical Research 49 (2016) 2015-2022.

[20] Q. Jia, D. E. Ramaker, J. M. Ziegelbauer, N. Ramaswamy, A. Halder, S. Mukerjee, The Journal of Physical Chemistry C 117 (2013) 4585-4596.

[21] L. W. H. Leung, T. W. Gregg, D. W. Goodman, Langmuir 7 (1991) 3205-3210.

[22] Z. Yu, J. Zhang, Z. Liu, J. M. Ziegelbauer, H. Xin, I. Dutta, D. A. Muller, F. T. Wagner, The Journal of Physical Chemistry C 116 (2012) 19877-19885.

[23] P. Jovanovič, V. S. Šelih, M. Šala, S. B. Hočevar, A. Pavlišič, M. Gatalo, M. Bele, F. Ruiz-Zepeda, M. Čekada, N. Hodnik, M. Gaberšček, Journal of Power Sources 327 (2016) 675-680.

[24] F. Ruiz-Zepeda, M. Gatalo, P. Jovanovič, A. Pavlišič, M. Bele, N. Hodnik, M. Gaberšček, ChemCatChem 9 (2017) 3904-3911.

[25] Y. Kang, J. Snyder, M. Chi, D. Li, K. L. More, N. M. Markovic, V. R. Stamenkovic, Nano Letters 14 (2014) 6361-6367.

[26] N. Hodnik, M. Bele, S. Hočevar, Electrochemistry Communications 23 (2012) 125-128.

[27] M. Bele, M. Gaberscek, G. Kapun, N. Hodnik, S. Hocevar, US9147885B2, 2015.

[28] D. van der Vliet, D.S. Strmcnik, C. Wang, V. R. Stamenkovic, N. M. Markovic, M. T. M. Koper, Journal of Electroanalytical Chemistry 647 (2010) 29-34.

[29] K. J. J. Mayrhofer, D. Strmcnik, B. B. Blizanac, V. Stamenkovic, M. Arenz, N. M. Markovic, Electrochimica Acta 53 (2008) 3181-3188. 
[30] M. Pourbaix, Atlas of Electrochemical Equilibria in Aqueous Solutionis, National Association of Corrosion Engineers1966.

[31] J. Snyder, I. McCue, K. Livi, J. Erlebacher, Journal of the American Chemical Society 134 (2012) 86338645.

[32] I. McCue, J. Snyder, X. Li, Q. Chen, K. Sieradzki, J. Erlebacher, Physical Review Letters 108 (2012) 225503.

[33] L. Dubau, J. Durst, F. Maillard, L. Guétaz, M. Chatenet, J. André, E. Rossinot, Electrochimica Acta 56 (2011) 10658-10667.

(C2018 by the authors; licensee IAPC, Zagreb, Croatia. This article is an open-access article distributed under the terms and conditions of the Creative Commons Attribution license (http://creativecommons.org/licenses/by/4.0/) 